\title{
OBTENTION DE NAISSANCES GÉMELLAIRES APRES SUPEROVULATION LIMITÉE ET CARACTÉRISTIQUES ZOOTECHNIQUES DES VACHES PRODUCTRICES ET DES VEAUX JUMEAUX
}

\author{
P. MAULEON, M.-J. BOSC, M. COUROT, J. PELOT, J. SCHNEBERGER et R. ORTAVANT \\ avec la collaboration technique de G. Covlaud et $Y$. DE Fontaubert \\ Institut national de la Recherche agronomique \\ Laboratoire de Physiologie de la Reproduction, 37 -Nouzilly, France \\ Domaine de Bressonvillers, 91 - Leudeville, France
}

Des naissances gémellaires ont été obtenues après traitement hormonal chez les Bovins depuis plus de trente ans (ZAWAdowsky et Eskin, I937). Mais, bien que HAmmond (I958) ait attiré l'attention de nombreux chercheurs sur l'intérêt d'une telle technique pour augmenter la production de viande bovine, aucune expérience n'a permis de dire quel niveau d'intensification de la production permettrait d'atteindre l'utilisation de la méthode hormonale de superovulation limitée. Ceci est explicable par le coût de telles expériences qui a entraîné une très grande dispersion d'essais à petite échelle (HAmmond Jr., I949 ; Petrov, Khari,AMPIDI et INDísskAYA, I960; Lo'T'T et GaLi, I96I ; ARBEITER, I962).

Gordon et al (I962) tentèrent néanmoins de faire une telle application de la méthode de superovulation chez les éleveurs. Ils conclurent que les problèmes techniques posés par l'utilisation de PMSG étaient trop nombreux pour qu'une recommandation pratique puisse être donnée ; cette position était sage tant était difficile de dire à la fin de cet essai, combien de veaux pourraient être produits par Ioo vaches ainsi traitées, plusieurs incidents ou erreurs de méthodologie étant venus troubler la réussite de cette technique.

Nous avons donc pensé qu'en utilisant une population bovine homogène et saine, nous devions et pouvions déterminer ce paramètre global indispensable pour juger de la valeur pratique du traitement hormonal défini antérieurement (chap. III). 


\section{MATÉRIEL ET MÉTHODES}

Les résuitats de trois expériences seront rapportés : deux ont été réalisées (expériences $\mathrm{D}_{\mathrm{I}}$ et $D_{\text {II }}$ ) respectivement en janvier-novembre I966 et en janvier-octobre I 968 sur un troupeau de I 00 vaches Française Frisonne Pie-Noire, au domaine de Bressonvillers près de Brétigny (Essonne). Ces vaches constituaient une population indemne de brucellose, de niveau de production laitière élevée; elles ont été traitées après leur première ou sixième mise-bas et entretenues en stabulation libre avec une alimentation constante toute l'année. La majeure partie des 30 animaux des résultats de l'expérience I968 (DII) avait déjà subi un traitement hormonal en I966.

Dans les deux cas, l'injection d'hormone gonadotrope sérique a été faite 4 jours avant la date présumée de l'œstrus et l'injection d'hormone gonadotrope chorionique le jour de l'œstrus ; l'œstrus était déterminé par observation du comportement sexuel entre femelles. La dose de HCG injectée a été dans tous les cas de I 500 UI. Én I966, 9I vaches ont reçu 2 ooo UI de PMSG et 7 animaux I 800 UI. En I968 la dose de PMSG a été ajustée en fonction du poids des animaux variant de I 500 UI pour les vaches de $400-45^{\circ} \mathrm{kg}$ à 2 ooo UI pour celles de poids supérieur à $700 \mathrm{~kg}$. Tous les animaux ont été saillis pendant toute la durée des chaleurs. Lorsque les vaches sont revenues en chaleurs, elles ont été saillies sans nouveau traitement hormonal jusqu'à établissement d'une gestation.

La troisième expérience (expérience B IV) s'est déroulée au C. R. V. Z. de Nouzilly dans les conditions précisées au chapitre VI. 58 vaches ont reçu le traitement hormonal de I 600 UI de PMSG + I 500 UI de HCG ; celles qui n'ont pas été gestantes ont reçu à nouveau le même traitement et en cas de retours en chaleurs après ce $2^{\mathrm{e}}$ traitement, les animaux ont été éliminés sans que la fertilité consécutive à ces deux traitements ait été déterminée. Il s'agit des mêmes animaux sur lesquels a été établie la corrélation entre le niveau de superovulation et l'obtention d'une gestation (chap. VI).

Les veaux des expériences $D_{I}$ et $D_{I I}$ ont été soumis à des conditions de production de veaux d'élevage et ceux de l'expérience B IV ont reçu une alimentation type veaux d'engraissement.

\section{RÉSULTATS}

\section{Niveau d'intensification de la production de veaux}

\section{Il peut se définir par rapport à quatre paramètres :}

- le nombre de veaux produits par Ioo vaches gestantes à la chaleur de superovulation ;

- le pourcentage de vaches gestantes après saillie aux chaleurs observées après traitement hormonal;

- le pourcentage de vaches présentant des retours tardifs (plus de 42 jours après la saillie) ;

- la fertilité ultérieure des vaches au cours des cycles de retours en chaleurs après leur remise en reproduction normale.

Les figures $I, 2$ et 3 précisent les trois premiers paramètres pour chacune des expériences $D_{1}, D_{I I}$ et $B_{I V}$.

Les nombres de veaux produits par Ioo vaches gestantes sont respectivement de $\mathrm{r}_{50}$ et de I40 pour les animaux traités à la ferme de Bressonvillers; ils sont de I 48 et de $\mathbf{3} 30$ pour les animaux de l'expérience $B_{I v}$ au premier et au deuxième traitement.

Les taux de gestation après ces chaleurs sont de 44,$9 ; 59,3$ et 53,4 p. Ioo ; ils sont évidemment le reflet de l'importance des retours tardifs qui ont été respectivement de $3 \mathrm{I}, 6 \mathrm{p}$. IOo ; I5,6 p. Ioo et $20,7 \mathrm{p}$. Ioo ; le taux de non-retours en chaleurs 


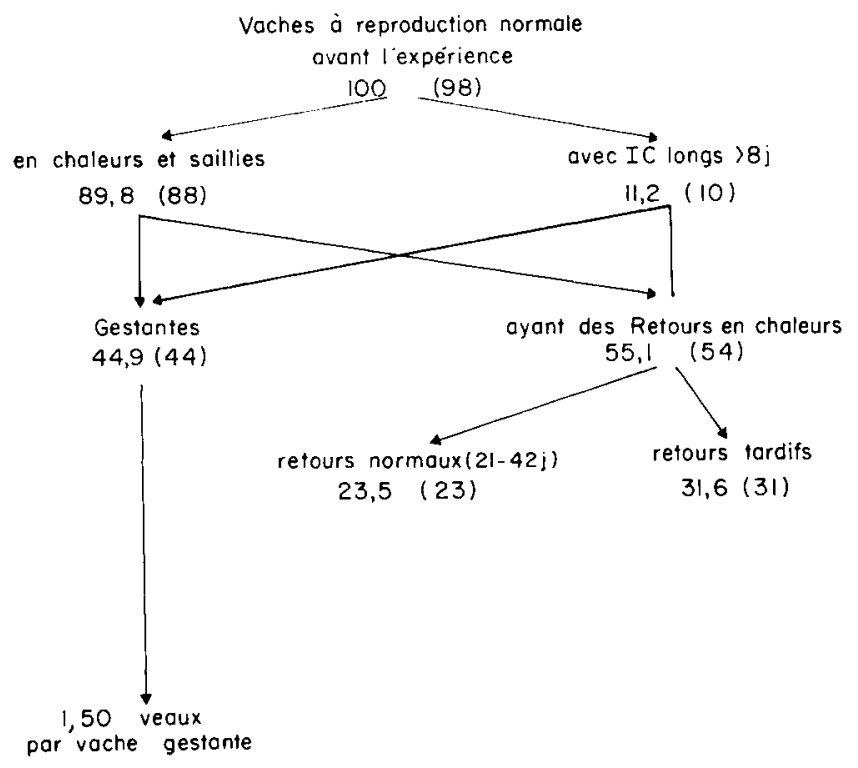

FIG. I. - Bilan de l'expérience, naissances gémellaires (Bressonvillers, I966)

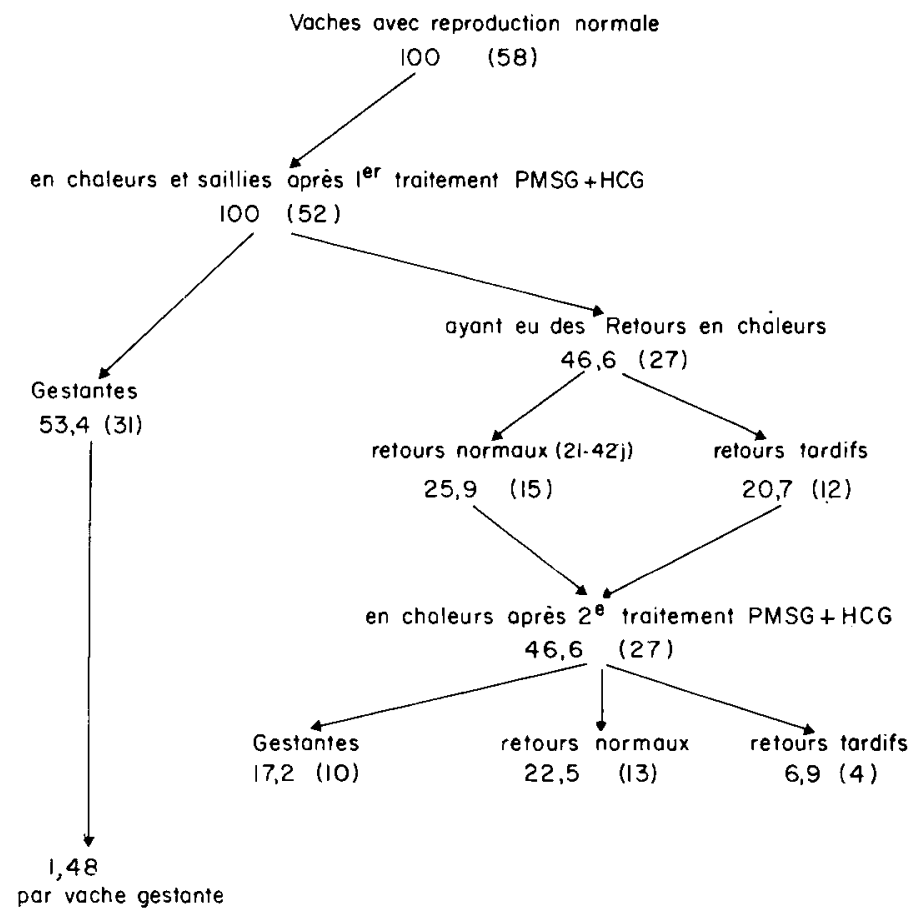

likg. 2. - Bilan établi d'après l'expérience $B_{J V}$

(Nouzilly, I968) 
à 42 jours est très voisin pour les trois groupes de vaches et compris entre 74,7 p. Ioo et 76,5 p. roo, c'est-à-dire excellent.

Mais le nombre de veaux produits par roo vaches est évidemment aussi la conséquence de la fertilité des cycles de retours en chaleurs et de la possibilité pour ces vaches de donner rapidement un veau. Nous ne possédons pas cette donnée pour les vaches de l'expérience $B_{I V}$. Par contre, tous les animaux traités appartenant au domaine de Bressonvillers ont eu un veau après ce traitement hormonal. Nous avons estimé cette fertilité en fonction du nombre de saillies nécessaires pour obtenir une nouvelle gestation.

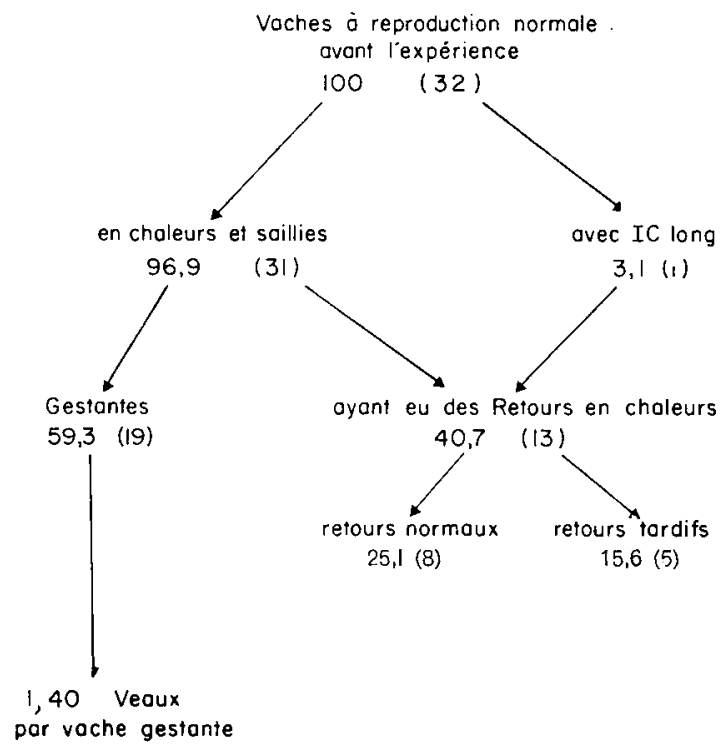

FIG. 3. - Bilan de l'expérience, naissances gêmellaives (Bressonvillers, I968)

Sur les 54 vaches non gestantes aux chaleurs dites "de superovulation ", 46 soit 85,2 p. Ioo ont été gestantes après 4 saillies au maximum (expérience $D_{\mathrm{I}}$ ) et II sur I2 soit $9 \mathrm{I}, 7 \mathrm{p}$. Ioo pour l'autre groupe de vaches (expérience $\mathrm{D}_{\mathrm{II}}$ ).

Nous avons donc obtent une production de I22,4 veaux pour roo vaches traitées (expérience $D_{I}$ ) et de I23,7 pour Ioo vaches traitées (expérience $D_{\text {II }}$ ), cette production ayant effectivement été réalisée en une année pour les vaches de cette dernière expérience.

Si les 7 vaches non gestantes après deux traitements de l'expérience $B_{I V}$ n'avaient pas été éliminées mais remises à la reproduction nous pourrions estimer que la réalisation de deux traitements successifs permettraient d'atteindre un niveau de production de I3I,I veaux pour Ioo vaches traitées.

2. Caractéristiques comparées des vaches ayant produit des veaux jumeaux et simples

La durée de la gestation est plus courte chez les vaches portant deux ou trois fœetus, une corrélation positive de $r=+0,53$ existant entre la durée de gestation et le poids des veaux nés simples (tabl, I). 
D'autre part, bien qu'il n'ait pas été fait de corrélation entre les productions successives d'un même animal avant, pendant et après l'obtention de jumeaux, il ne semble pas d'après les valeurs moyennes présentées dans le tableau 2 que la production laitière ait été affectée; il faut remarquer cependant que la production des vaches ayant donné des triplés est plus faible que celle des animaux ayant donné des jumeaux.

\section{TABLEAU I}

Durée de gestation des vaches ayant mis-bas un, deux ou trois veaux

(Expérience $D_{I}$, Bressonvillers, I966)

\begin{tabular}{|c|c|c|c|c|c|c|}
\hline Type de gestation & $\begin{array}{c}\text { Durée de } \\
\text { gestation (j) } \\
\bar{m} \pm \mathrm{Sm}\end{array}$ & $\begin{array}{c}\text { Nombre } \\
\text { animaux }\end{array}$ & $\begin{array}{c}\text { Poids moyen } \\
\text { veau (kg) } \\
m \pm \mathrm{Sm}\end{array}$ & $\begin{array}{c}\text { Nombre } \\
\text { animaux }\end{array}$ & $\begin{array}{l}\text { Poids moyen } \\
\text { mère (kg) } \\
m \pm \mathrm{Sm}\end{array}$ & $\begin{array}{l}\text { Nombre } \\
\text { animaux }\end{array}$ \\
\hline Simple .. & $276,0 \pm 0,7$ & 59 & $39,3 \pm 0,6$ & 59 & $661 \pm 10$ & 35 \\
\hline Double... & $270,5 \pm 1,4$ & 8 & $31,5 \pm 0,7$ & 16 & $606 \pm 20$ & 8 \\
\hline Triple.......... & $260,8 \pm 4,5$ & 5 & $22,5 \pm 0,8$ & 15 & $614 \pm 22$ & 5 \\
\hline
\end{tabular}

TABLEAU 2

Production laitière des vaches ayant mis-bas des veaux doubles et triples (en $\mathrm{kg}$ de lait pendant $27^{\circ}$ jours) - Expérience DI, Bressonvillers, I964

\begin{tabular}{|c|c|c|c|c|c|c|c|}
\hline \multirow{2}{*}{$\begin{array}{l}\text { No de } \\
\text { lactation }\end{array}$} & \multirow{2}{*}{$\begin{array}{c}\text { Production } \\
\text { moyenne avant } \\
\text { naissances } \\
\text { multiples }\end{array}$} & \multicolumn{2}{|c|}{$\begin{array}{c}\text { Production moyenne } \\
\text { pendant gestation } \\
\text { multiple }\end{array}$} & \multicolumn{2}{|c|}{$\begin{array}{l}\text { Production moyenne } \\
\text { après } \\
\text { naissances multiples }\end{array}$} & \multicolumn{2}{|c|}{$\begin{array}{l}\text { Production moyenne } \\
\text { aux } \\
\text { lactations suivantes }\end{array}$} \\
\hline & & Doubles & Triples & Doubles & Triples & Doubles & Triples \\
\hline$I$ & $3657 \quad(10)$ & $3524 \quad(6)$ & 3857 (1) & 4415 & - & - & - \\
\hline II & $4267 \quad(14)$ & - & 3198 (1) & 4709 & $4332 \quad(4)$ & - & - \\
\hline III & $5112 \quad(13)$ & 4977 & 4467 & - & 4230 (1) & 一 & 6039 (1) \\
\hline IV & 5633 & $5972 \quad(1)$ & - & +986 & $4708(3)$ & 一 & 3690 (1) \\
\hline $\mathrm{V}$ & $57 r_{4} 6$ & $5813 \quad(5)$ & 5393 (1) & 6042 & $\longrightarrow$ & 5235 (1) & $5008 \quad(3)$ \\
\hline VI & 6111 & $6111 \quad(1)$ & $一$ & 5821 & 6237 (1) & - & - \\
\hline VII & - & - & - & 4850 & - & $6013(5)$ & $6693(1)$ \\
\hline Moyenne & $4813 \quad\left(5 y^{\prime}\right)$ & $4816(15)$ & $4157(9)$ & $5195(15)$ & $4657(9)$ & $5908(6)$ & $5211 \quad(6)$ \\
\hline
\end{tabular}

(Nombre de vaches considérées dans les moyennes).

3. Caractéristiques de croissance des veaux nés doubles et triples (tabl. 3, 4)

Les résultats rapportés sur la croissance de 22 veaux nés doubles et de II veaux nés triples montrent qu'en dépit d'un handicap de poids à la naissance par rapport à ceux nés simples, une croissance quotidienne comparable après le sevrage leur permet d'atteindre un poids voisin à un an. 

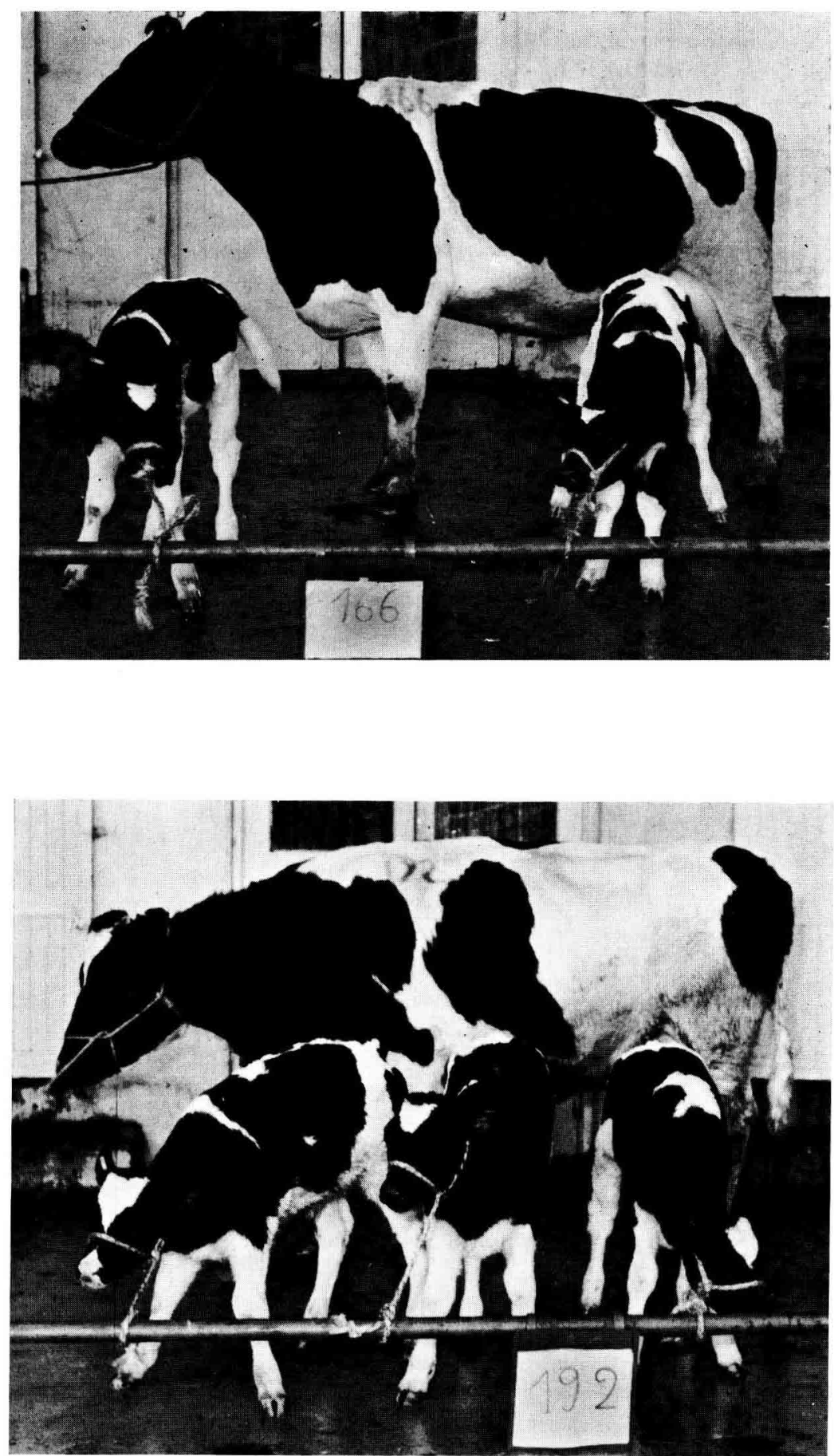

Protos 1 et 2. - Un exemple de veaux nes doubles ou triples it la swite dit traitement PMSG + $H C G$ 
Le poids total de veau âgé de 3 mois et demi à 4 mois vendu par vache a été de $263,7 \mathrm{~kg}$ et $357 \mathrm{~kg}$ pour celles ayant donné respectivement des jumeaux ou des triplés. Les vaches ayant donné des simples ont permis de vendre $126,7 \mathrm{~kg}$ de veau. Ainsi malgré des conditions d'élevage défavorables dans ces différentes expériences les résultats montrent cependant les importantes possibilités de la méthode.

\section{TABI,EAU 3}

Croissances comparées des veaux nés simples, doubles et triples (Expérience Di, Bressonvillers, 1966)

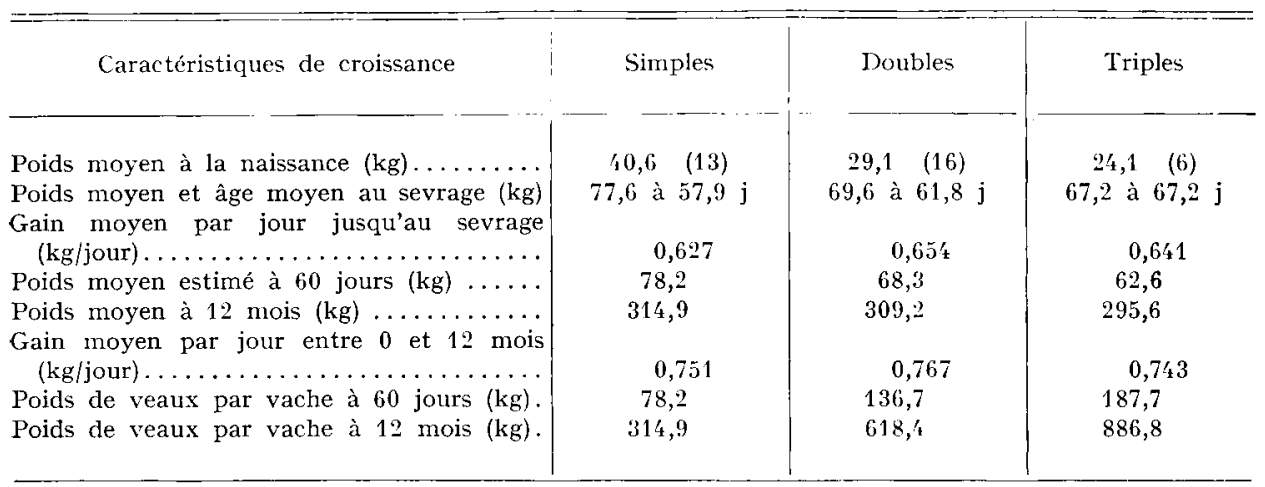

( ) Nombre d'animaux.

\section{TABLEAU 4}

Croissances comparées des veaux nés simples, doubles et triples

(Expérience $\mathrm{B}_{\mathrm{IV}},{ }^{\mathrm{re}}$ partie, Nouzilly, I968)

\begin{tabular}{|c|c|c|c|}
\hline Caractéristiques de croissance & Simples & Doubles & Triples \\
\hline $\begin{array}{l}\text { Poids moyen à la naissance } \ldots \ldots \ldots \ldots \ldots \ldots \\
\text { Poids moyen à } 60 \text { jours } \ldots \ldots \ldots \ldots \ldots \ldots \ldots \\
\text { Poids moyen à } 90 \text { jours } \ldots \ldots \ldots \ldots \ldots \ldots \ldots \\
\text { Poids et âge moyen à la vente }(\mathrm{kg}) \ldots \ldots \ldots \\
\text { Poids total de veaux par vache (kg) au } \\
\text { moment de la vente (106 à } 118,8 \text { jours).. }\end{array}$ & $\begin{array}{c}33,62 \quad(13) \\
70,50 \quad(12) \\
107,50(12) \\
126,67 \stackrel{\text { à }}{106,00 \mathrm{j}} \\
126,67\end{array}$ & $\begin{array}{c}21,37 \quad(8) \\
6, t, 66 \quad(6) \\
99,50 \quad(6) \\
131,83 \text { à } 115,00 \mathrm{j} \\
263,66\end{array}$ & $\begin{array}{c}21,89 \quad(9) \\
5 \prime, 80 \quad(5) \\
86,40 \quad(5) \\
119,00 \text { à } 118,80: \\
357,00\end{array}$ \\
\hline
\end{tabular}

\section{DISCUSSION}

Les résultats obtenus au cours de ces trois expériences définissent un niveau de production possible situé entre I23 et I3o veaux par vache traitée et par an. Ce niveau peut être atteint si le pourcentage de vaches ayant des ovulations silencieuses est faible (cas des expériences $D_{I I}$ et $B_{I V}$ ). Ceci est possible grâce à une bonne connaissance du cycle œstrien des vaches. Celle-ci a été insuffisante au cours de la première 
expérience réalisée au domaine de Bressonvillers et elle est vraisemblablement la cause des I4,3 p. Ioo d'ovulations silencieuses de l'expérience anglaise (GoRdon et al., I962) (fig. 4) et des 69 p. roo de vaches non venues en ostrus rapportées dans les dernières expériences russes (KATSY et VoLKOV, I968).

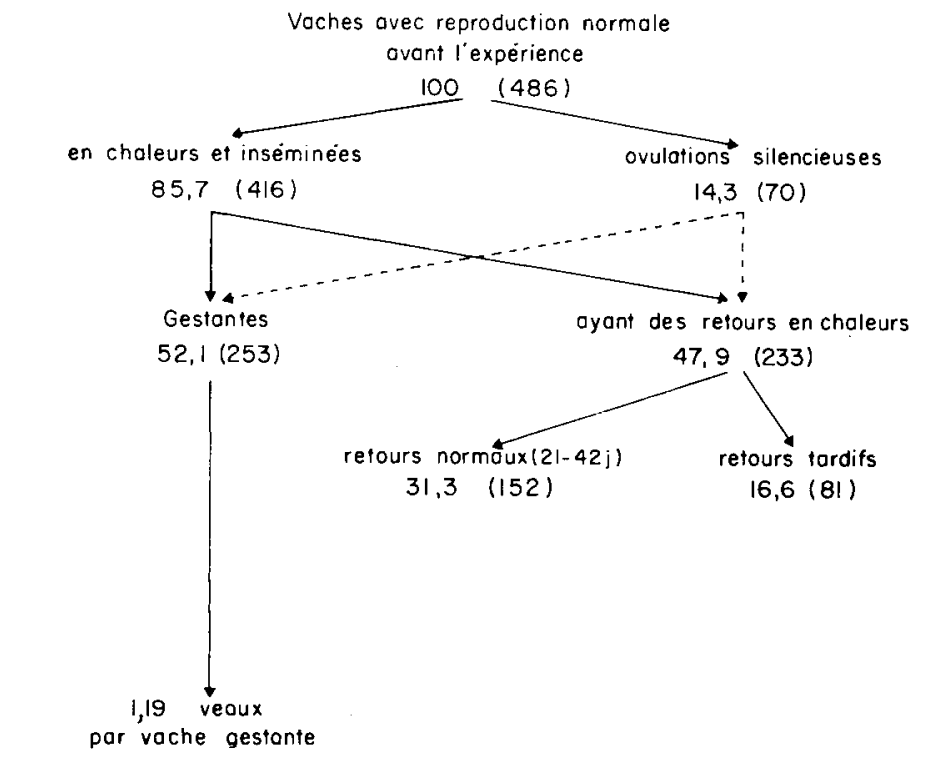

FIc. 4. -- Bilan ćtabli d'apris l'expéricnce rapportée par GoRdon ct al. (I962) (entre parenthèses : nombre de vaches)

Une telle production n'est réalisable que si le nombre de veaux par vache gestante après superovulation est de l'ordre de I,40 à I,50. GoRdon et al. (I962) en utilisant plusieurs doses de PMSG ne pouvaient pas, en obtenant seulement $\mathrm{I}, 20$ veau par vache gestante après superovulation, espérer plus de IIo veaux par roo vaches traitées. La figure 4 a été établie d'après les résultats de ces auteurs en supposant que les vaches pour lesquelles il y a eu un contrôle du nombre de fotus par éclatement de la poche amniotique auraient donné le même résultat que les autres vaches de l'expérience.

Le taux de production de veaux par vache gestante augmente avec la proportion de vaches ayant superovulé. Mais l'accroissement de cette proportion s'accompagne aussi, fréquemment, d'une augmentation du pourcentage de vaches ayant plus de 4 ovulations, c'est-à-dire d'une augmentation des retours tardifs. Plusieurs méthodes semblent néanmoins possibles pour diminuer cette proportion :

- soit effectuer deux traitements successifs de PMSG + HCG comme dans l'expérience $B_{I V}$; dans ce cas, la proportion d'animaux avec un nombre élevé d'ovulations diminue au deuxième traitement;

- soit injecter deux doses modérées de PMSG à deux moments du même cycle œstrien selon la méthode de Schri.ring et HoL.M (I963).

L'utilisation de cette dernière méthode vient de permettre à Turmax et al. (I 969) d'obtenir I32,7 veaux par vache gestante après le traitement de superovulation sur des vaches Hereford. Ceci correspond à environ I,20 veau par vache traitée. 
- Soit essayer de "personnaliser " la dose d'hormone injectée : c'est-à-dire adapter celle-ci à la sensibilité de chaque animal. C'est sans doute 1'une des voies d'avenir.

\section{CONCLUSION}

En s'appuyant sur des résultats obtenus sur I 86 vaches Française Frisonne PieNoire, il a été possible d'obtenir I,40 à I,50 veau par vache gestante à la suite d'une saillie lors de l'œestrus de superovulation. Ce résultat correspond à une production de I,23 à I,30 veau par vache traitée. Cet objectif est actuellement accessible à condition que le niveau de superovulation soit contrôlé de façon à maintenir la proportion de retours tardifs à un niveau situé entre $I_{5}$ et $\mathrm{r} 8 \mathrm{p}$. Ioo, sans diminuer la proportion de vaches superovulées et que le contrôle des cycles astriens soit suffisant pour permettre la suppression de la presque totalité des ovulations silencieuses.

\section{RÉSUMÉ}

Afin de connaître combien de naissances gémellaires résulteraient d'une superovulation limitée provoquée par l'injection de l'hormone gonadotrope sérique, 4 jours avant la date présumée de l'cstrus et d'une injection intraveineuse d'hormone gonadotrope chorionique le jour de l'cestrus, ce traitement hormonal a été appliqué à 186 vaches de race Française Frisonne Pie Noire dont on a laissé les gestations se poursuivre jusqu'à leur terme.

La close de 2 ooo UI de PMSG injectée à 98 de ces animaux s'est révélée trop forte puisque 3I,6 p. Ioo des vaches sont revenues en chaleurs après 80 jours de gestation. Néanmoins il a été obtenu I,5 veaux par vache gestante et le nombre de veaux pour Ioo vaches traitées a atteint 122,4 .

En diminuant la dose de PMSG injectée à I 6oo UI et en traitant à nouveau de la même façon les vaches non gestantes, le nombre de veaux par ioo vaches traitées peut atteindre I3I. L'ajustement de la dose de PMSG injectée au poids des animaux diminue les retours tardifs à I5,6 p. roo et a permis de maintenir le nombre de veaux par vache gestante à 1,4 .

Les poids totaux de veaux âgés de 3 mois et demi à 4 mois produits par vache ont été de $263.7 \mathrm{~kg}$ et de $357 \mathrm{~kg}$ respectivement pour les animaux ayant mis-bas des doubles ou des triples et $126,7 \mathrm{~kg}$ pour ceux ayant mis-bas des simples.

\section{SUMMARY}

\section{TWIN BIRTHS AFTER LIMITED SUPEROVULATION. ANIMAL PRODUCTION CHARACTERISTICS OF THE COWS AND THEIR TWIN CALVES}

An experiment was performed to find out how many twin births may be obtained from a limited superovulation induced by the injection of PMSG four days before the presumed date of cestrus, and an intravenous injection of I,500 I. U. of HCG on the day of cestrus. I 86 French Friesian cows were treated, and their pregnancies allowed to develop to full-term.

The dose of $2,000 \mathrm{I}$. U. of PMSG administered to 98 of these animals was too strong because 31.6 p. I oo of the cows returned to ostrus more than 80 days after mating. Nevertheless, I.5 calves per pregnant cow were obtained, and the number of calves born per roo treated cows rose to I 22.4 . 
By decreasing the PMSG dose to $1,600 \mathrm{I}$. U., and repeating the same treatment on the nonpregnant cows, the number of calves per Ioo treated cows may reach I3I. The adjustment of the injected PMSG dose to the live weight of the animal decreased the delayed returns to 15.5 p. Ioo, and kept the number of calves per pregnant cow at I.4.

The total weights of 3.5-4 months old calves produced per cow were $263.7 \mathrm{~kg}$ and $357 \mathrm{~kg}$, respectively, for the twin or triplet animals, and 126.7 for the single.

Except for cows having given birth to triplets, it does not seem that milk yeld was affected by twin births.

\section{RÉFÉRENCES BIBLIOGRAPHIQUES}

Arbeiter K., I962. Vorlänfinger Bericht über Versuche zur hormonalen erzeugung non zwillingen bei Kühen. Wien. Tierärztl. Mschr., 49, I6I-I68.

Gordon I., Willians C., Edwards J., 1962. The use of serum gonadotrophin (PMSG) in the induction of twin pregnancy in the cow. J. Agric. Sci., 59, I43-199.

Hammond J., Bhattacharya P., i944. Control of ovulation in the cow. J. Agric. Sci., 34, I-15.

Hammond J., Jr., x949. Induced twin ovulations and multiples pregnancy in cattle. J. Agric. Sci., 39, 222-225.

Hammond J., 1958. Research in the physiology of reproduction. Agric. Res., 4, 17-20.

Katsy G. D., Volkov S. A., I 668 . Polyovulation et vêlages des vaches au cours de l'utilisation com. plexe du sérum de jument gravide et de progestérone (russe). Zhivotnovodstvo, 30, 83-84.

Lotri L., Galli S., I96r. I parti plurimi nella specie bovine. III. Prove praticle. Riv. Zootec., 34, 3I0-3II.

Petrov V. A., Harlampidi G. P., Inditskaya J. Y., i 960 . Essai de stimulation de la superovulation chez les vaches à l'aide d'une préparation de sérum de jument gravide. Zhivotnovodstvo, 22, 86-87.

Schilling E., Holm W., I963. Investigations on induction of limited multiple ovulations in cattle J. Reprod. Fert., 5, 283-286.

Turman E. J., Renbarger R. E., Stephens D. F., ig69. Producing multiple births in beef cows by hormone injections (non publié).

Zavadovsky M. M., Eskin I. A., I939. L'utilisation du polan et du SJG pour le contrôle du cycle sexuel chez la Vache (en russe). Trud. Dinam. Rastit., 11, I I2-132. 